\title{
MICROWAVE ASSISTED IMPROVED METHOD FOR THE SYNTHESIS, CHARACTERIZATION OF 1-(2-HYDROXY PHENYL)-METHANONE-3,5-DISUBSTITUTED PYRAZOLINES
}

\author{
Shrikant A. Wadhal ${ }^{1, *}$ and Imran Khan ${ }^{2}$ \\ Department of Chemistry, Shri Shivaji Science College, Amravati (M.S.) - 444603, \\ *E-mail:shrikrish123@gmail.com
}

\begin{abstract}
The present research has systematic approach to synthesized a series of 1- pyridine-4-yl-methanone-3,5disubstituted Pyrazolines derivatives by the action of Methyl Salicylate and1-(4-methyl phenyl)-3-(phenyl)-prop-2ene-1-one. In the present study, an attempt has been made to synthesize compounds by green chemistry technique i.e. microwave synthesis which is more convenient than conventional method Structures of all the synthesized compounds were confirmed by their IR, ${ }^{1} \mathrm{H}-\mathrm{NMR}$.
\end{abstract}

Keywords: Pyrazolines, 1,3-Disubstituted-prop-2-ene-1-one, Methyl Salicylate, MWI.

(C) RASĀYAN. All rights reserved

\section{INTRODUCTION}

Pyrazolines derivatives have been extensively documented because of their broad spectrum biological activities. The pharmaceutical importance of these compounds lies in the fact that they can be effectively utilized as analgesic ${ }^{1}$, antioxidant ${ }^{2}$, antidepressant ${ }^{3}$, anticancer ${ }^{4}$ and antidiabetic agents ${ }^{5}$, immunosuppressive effect ${ }^{6}$ and antitumor activity ${ }^{7}$ of some pyrazolines derivatives are also known.

Microwave $^{8-13}$ has been applied in science research as an assistant technique or a method to chemical synthesis. It may be considered as a green chemistry technique which focuses on more efficient and faster reaction than conventional method. Because of structural and packing effects, reaction products may be obtained in solid state. The microwave based approaches have been introduced gradually and played an important role in the process of preparation. Compared to the traditional heating methods, the microwave treatment provides intensive, homogeneous and efficient energy, and thus it can achieve the elevated temperature and initiate the reaction in an extremely short time.

In the present study, an attempt has been made to synthesize compounds by green chemistry technique i.e. microwave synthesis which is more convenient than conventional method ${ }^{14}$. The structures of synthesized compounds were confirmed by spectral analysis.

\section{EXPERIMENTAL}

General Conditions: Melting points are uncorrected and were determined in open capillary tubes in. TLC was performed on silica gel-G and spotting was done using iodine. IR spectra were recorded on Nicolet 5ZDXFT-IR spectrometer in KBr phase and ${ }^{1} \mathrm{HNMR}$ on Brucker WP 200 and $500 \mathrm{SY}$.

\section{General Procedure}

Preparation of 1-(2-hydroxy phenyl) methanon-3-(4-methyl phenyl)-5-(phenyl) pyrazoline (1C)

A mixture of $1 \mathrm{~A}(0.005 \mathrm{M})$ and $1 \mathrm{~B}(0.005 \mathrm{M})$ were mixed together in an Erlenmeyer flask in microwave oven and irradiated with microwave radiation for 2 minutes by keeping wattage knob at 160 watt and times knob at 2 minutes. After completion of reaction mixture was cooled. The completion of reaction was determined by TLC. The crude product obtained was recrystallized from ethanol to get pure compound (1C). 
RASĀYAN J. Chem.

Vol. 10 | No. 2 |630 - 633 | April - June | 2017<smiles>Cc1ccc(C(=O)/C=C/c2ccccc2)cc1</smiles><smiles>NNC(=O)c1ccccc1O</smiles><smiles></smiles>

(1B)

Scheme-1

\section{RESULTS AND DISCUSSION}

Reaction of 1,3-disubstituted-prop-2-ene-1-one 2 (a-e ) with methyl salicylate on microwave irradiation gives 1-(2-hydroxy phenyl)-4-yl-methanone-3,5-disubstituted Pyrazolines (3a-e). The structures of synthesized compounds were characterized on the basis of its spectral data. Thus, its IR spectrum in $\mathrm{KBr}$, showed a strong peak at $1632 \mathrm{~cm}^{-1}$ due to $\mathrm{C}=\mathrm{O}$ group, $3341 \mathrm{~cm}^{-1}$ due to $-\mathrm{OH}$ group in pyrazoline, 
${ }^{1} \mathrm{HNMR}$ spectrum showed three characteristic double doublets of $(\mathrm{H} 1, \mathrm{H} 2, \mathrm{H} 3)$ protons of pyrazolines. Microwave assisted synthesis is more convenient with better yield than conventional method.

Table 1: The IR-Spectra and ${ }^{1} \mathrm{H}-\mathrm{NMR}-$ Spectra of (1C) Compound

\begin{tabular}{c|c|c|c}
\hline S. No. & Class of Compound & Types of Vibration & Frequency in $\mathrm{cm}^{-1}$ \\
\hline 1. & Aromatic $-\mathrm{OH}$ & $3341 \mathrm{~cm}^{-1}$ \\
\hline 2. & $\mathrm{C}=\mathrm{O}$ stretch & $1672 \mathrm{~cm}^{-1}$ \\
\hline 3. & Imines & P- subst. Ar-ring & \\
\hline 4. & Mono subst. Ar-ring & $829 \mathrm{~cm}^{-1}$ \\
\hline 5. & &
\end{tabular}

Table-2: Physical data of synthesized compound

\begin{tabular}{c|c|c|c}
\hline S. No. & Chemical Shift $(\delta)$ in ppm & Multiplicity & Correlation \\
\hline 1. & $3.2-3.4 \mathrm{ppm}$ & Singlet & $3 \mathrm{H}-\mathrm{CH}_{3}$ \\
\hline 2. & $3.4-3.5 \mathrm{ppm}$ & Double doublet & $\mathrm{H}_{\mathrm{A}}$ \\
\hline 3. & $3.6-3.8 \mathrm{ppm}$ & Double doublet & $\mathrm{H}_{\mathrm{B}}$ \\
\hline 4. & $4.7-4.84 \mathrm{ppm}$ & Double doublet & $\mathrm{H}_{\mathrm{X}}$ \\
\hline 5. & $6.8-8.1 \mathrm{ppm}$ & Multiplet & $13 \mathrm{H} \mathrm{Ar}-\mathrm{H}$ \\
\hline 6. & $10.2 \mathrm{ppm}$ & Broad singlet & $\mathrm{H}, \mathrm{OH}$ \\
\hline
\end{tabular}

Table-3: Melting Points and Yields of Synthesized Compounds

\begin{tabular}{|c|c|c|c|c|}
\hline S.No. & Compound Name & M.P. & Yield & Time \\
\hline 1. & $\begin{array}{l}\text { 1-(2-hydroxy phenyl) methanon-3-(4-methyl phenyl)-5- } \\
\text { (4-methoxy phenyl) pyrazoline.(1a) }\end{array}$ & $120^{\circ} \mathrm{C}$ & $83 \%$ & 1:30 Min \\
\hline 2. & $\begin{array}{l}\text { 1-(2-hydroxy phenyl) methanon-3-(4-methyl phenyl)-5- } \\
\text { (furyl) pyrazoline (1b) }\end{array}$ & $135^{\circ} \mathrm{C}$ & $87 \%$ & $1: 30 \mathrm{Sec}$ \\
\hline 3. & $\begin{array}{l}\text { 1-(2-hydroxy phenyl) methanon-3-(4-methyl } \\
\text { phenyl)-5-(phenyl) pyrazoline.(1c) }\end{array}$ & $125^{\circ} \mathrm{C}$ & $84 \%$ & $2 \mathrm{Min}$ \\
\hline 4. & $\begin{array}{l}\text { 1-(2-hydroxy phenyl) methanon-3-(4-methyl phenyl)-5- } \\
\text { (4-chloro phenyl) pyrazoline.(1d) }\end{array}$ & $308^{\circ} \mathrm{C}$ & $87 \%$ & $1: 30 \mathrm{Sec}$ \\
\hline 5. & $\begin{array}{l}\text { 1-(2-hydroxy phenyl) methanon-3-(4-methyl phenyl)-5- } \\
\text { (4-methyl phenyl) pyrazoline.(1e) }\end{array}$ & $128^{\circ} \mathrm{C}$ & $82 \%$ & $2 \mathrm{Min}$ \\
\hline
\end{tabular}

\section{ACKNOWLEDGMENT}

The authors would like to thanks authorities of Shri Shivaji Science College, Amravati University, Amravati for providing necessary facilities. 


\section{REFERENCE}

1. R.H. Udapi, Narayan Rao, A.R. Bhat, Indian J Heterocyclic Chemistry, 7, 217(1998)

2. P. Venkatesh ., HariPrasath, S. Sharfudeen , V. Soumya , V. Spandana, Priyanka, J. Pharm. Res., 5(5), 2875 (2012)

3. E. Palaska, M. Aytemir, T. Uzbay, Erol Dilek, Eur. J. Med. Chem.,36,539(2001)

4. F. Manna, F. Chimenti, R. Fioravanti, A. Bolasco, D. Seecci, P. Chimenti., Ferlini, Scambia G., Bioorg. Med.chem. Lett.,15, 4632(2005).

5. J. H. Ahn, H.M. Kim, S.H. Jung, S.K. Kang, K.R. Kim, S.D. Rhee, S.D. Yang, H.G. Cheon ,S.S.Kim, Bioorg. Med. Chem. Lett., 14, 4461(2004)

6. J.G. Lombardino, I.G. Otterness, J. Med. Chem., 20, 830(1977).

7. E.C. Taylor, H. Patel, H. Kumar, Tetrahedron, 48, 8089(1992).

8. S.A. Wadhal, Indo American J. of Pharmaceutical Research, 4(11),1462(2014).

9. B.P.Nandeshwarappa, D.B.Aruna Kumar, H. S. Bhojya Naik, V. P. Vaidya and K. M.Mahadevan, Indian J. Chem., 44, 2155(2005).

10. S. Ailwadi, Jyoti, Yadav, M. Pathak, D. Der. Pharma. Chemica., 3, 215(2011).

11. R.S. Talegaonkar, A.S. Burghateand S.A.Wadhal, Indian J. Heterocyclic Chem., 20, 413(2011).

12. Ankush Wakode, Archana Burghate, Shrikant Wadhal, Indo American Journal of Pharmaceutical Research,10, 5010(2014).

13. A. Loupy,A.Petit, TesierBoullet, Jacquate P., Mathe D, Synthesis, 9,1213(1998).

14. S. A. Wadhal, K.N. Wadodkar, P. S. Pande, Indian J. Heter. Chem., 15, 11(2005).

[RJC-1666/2017] 